\title{
Communication network formation with link specificity and value transferability
}

Citation for published version (APA):

Harmsen - van Hout, M. J. W., Herings, P. J. J., \& Dellaert, B. G. C. (2013). Communication network formation with link specificity and value transferability. European Journal of Operational Research, 229(1), 199-211. https://doi.org/10.1016/j.ejor.2013.02.028

Document status and date:

Published: 16/08/2013

DOI:

10.1016/j.ejor.2013.02.028

Document Version:

Publisher's PDF, also known as Version of record

Document license:

Taverne

Please check the document version of this publication:

- A submitted manuscript is the version of the article upon submission and before peer-review. There can be important differences between the submitted version and the official published version of record.

People interested in the research are advised to contact the author for the final version of the publication, or visit the DOI to the publisher's website.

- The final author version and the galley proof are versions of the publication after peer review.

- The final published version features the final layout of the paper including the volume, issue and page numbers.

Link to publication

\footnotetext{
General rights rights.

- You may freely distribute the URL identifying the publication in the public portal. please follow below link for the End User Agreement:

www.umlib.nl/taverne-license

Take down policy

If you believe that this document breaches copyright please contact us at:

repository@maastrichtuniversity.nl

providing details and we will investigate your claim.
}

Copyright and moral rights for the publications made accessible in the public portal are retained by the authors and/or other copyright owners and it is a condition of accessing publications that users recognise and abide by the legal requirements associated with these

- Users may download and print one copy of any publication from the public portal for the purpose of private study or research.

- You may not further distribute the material or use it for any profit-making activity or commercial gain

If the publication is distributed under the terms of Article $25 \mathrm{fa}$ of the Dutch Copyright Act, indicated by the "Taverne" license above, 
Decision Support

\title{
Communication network formation with link specificity and value transferability
}

\author{
Marjolein J.W. Harmsen - van Hout ${ }^{\text {a,* }}$, P. Jean-Jacques Herings ${ }^{\text {b, } 1}$, Benedict G.C. Dellaert ${ }^{c, 2}$ \\ a Institute for Future Energy Consumer Needs and Behavior (FCN), School of Business and Economics / E.ON Energy Research Center, RWTH Aachen University, Mathieustraße 10, \\ 52074 Aachen, Germany \\ ${ }^{\mathrm{b}}$ Department of Economics, Maastricht University, P.O. Box 616, 6200 MD Maastricht, The Netherlands \\ ${ }^{\mathrm{c}}$ Department of Business Economics / Marketing Section, Erasmus School of Economics, Erasmus University Rotterdam, P.O. Box 1738, 3000 DR Rotterdam, The Netherlands
}

\section{A R T I C L E I N F O}

\section{Article history:}

Received 27 April 2012

Accepted 15 February 2013

Available online 27 February 2013

\section{Keywords:}

Bilateral communication links

Link specificity

Value transferability

Social vs. informational value

Strategic network formation

\begin{abstract}
A B S T R A C T
We model strategic communication network formation with (i) link specificity: link maintenance lowers specific attention and thus value (negative externality previously ignored for communication) and (ii) value transferability via indirect links for informational but not for social value (positive externality modeled uniformly before). Assuming only social value, the pairwise stable set includes many nonstandard networks under high and particular combinations of complete components under low link specificity. Allowing for social and informational value reduces this set to certain fragmented networks under high and the complete network under low link specificity. These extremes are efficient, whereas intermediate link specificity generates inefficiency.
\end{abstract}

(c) 2013 Elsevier B.V. All rights reserved.

\section{Introduction}

In this paper we study the structure of bilateral communication links as decentrally emerging among individuals. Such structures of who communicates with whom can be identified in many important contexts, e.g., Trier (2008) visualizes digital communication networks like the increasingly popular virtual communities, and Hancock and Raeside (2010) analyze communication patterns in the operation management of a complex service process, and they determine important outcome variables such as the extent to which value is shared throughout a community and how it is distributed (e.g., Granovetter, 2005, Galeotti, 2010). Although the OR literature has a strong record of optimizing the central design of communication networks (e.g., Campbell, 1994; Kelly et al., 1998), decentrally developing networks have not received much attention in this field, exceptions being Baron et al. (2006), Monsuur (2007), and Janssen and Monsuur (2012). We model their formation as a game-theoretic network formation process in which agents face benefits and costs from being connected by a communication link and based on these two-sidedly decide on creating

\footnotetext{
* Corresponding author. Tel.: +49 2418049 835; fax: +49 2418049829.

E-mail addresses: mharmsen@eonerc.rwth-aachen.de (M.J.W. Harmsen - van Hout), p.herings@maastrichtuniversity.nl (P. Jean-Jacques Herings), dellaert@ese. eur.nl (B.G.C. Dellaert).

1 Tel.: +31 433883 824/636; fax: +31 433884878 .

2 Tel.: +31 104081 353; fax: +31 104089169 .
}

and maintaining a link. ${ }^{3}$ Using our model, we predict which stable communication networks emerge from the formation process and look at their efficiency properties. Hereby, we focus on a combination of two important aspects common to communication networks that has not been investigated before in the broad literature on strategic network formation: link specificity and the distinction between social and informational value.

First, our model features link specificity in the sense that the more direct connections an individual has to maintain with other individuals, the less she is able to specify her attention per link. Therefore, her value per link for others declines and she also derives less value from each link with others (cf. co-author model Jackson and Wolinsky, 1996). Thus, this feature is a negative network externality (e.g., Asvanund et al., 2004) and we go beyond the standard assumption of a fixed cost per link in communication networks (e.g., Bala and Goyal, 2000; connections model Jackson and Wolinsky, 1996). We assume that two connected agents contribute to their bilateral process of communication value creation according to a standard Cobb-Douglas production function with as inputs the amount of time invested by each agent in the link. Higher link specificity implies higher output elasticities in each bilateral value production process and therefore lower advantage of being connected with

\footnotetext{
${ }^{3}$ By contrast, Baron et al. (2006) is about information networks with one-sided link formation rather than communication networks with two-sided link formation, whereas Monsuur (2007) and Janssen and Monsuur (2012) consider only structural network characteristics as influencing the formation process rather than costs and benefits of being connected.
} 
several agents. Unit output elasticities are adopted to analyze high link specificity, while constant returns to scale, i.e., both output elasticities equal to $1 / 2$, reflect low link specificity.

Second, we introduce the important distinction between social and informational value as motivations for bilateral exchange decisions. This typology was suggested by the virtual community literature regarding the question why individuals choose to participate in and contribute to such a community as a whole (e.g., Mathwick et al., 2008). Social value is related to the fact that individuals may enjoy communicating with others, for example because they find it entertaining or because they feel it enhances their self-worth. Informational value refers to the fact that individuals may obtain new valuable knowledge from others when they communicate. Typically, informational value can be transferred relatively easily to third parties through indirect links, whereas social value is more personal and therefore hardly transferable without creating a direct link. To understand the relative impact of social and informational member orientation, we assume that social value is only experienced from direct neighbors and that informational value flows via any path consisting of bilateral communication links connecting two agents. Thus, the transferability of informational value is a positive network externality (e.g., Asvanund et al., 2004). Hereby, we integrate transferable and nontransferable value in one model, while so far, value transferability was at best incorporated uniformly for all value (e.g., Bala and Goyal, 2000; connections model Jackson and Wolinsky, 1996).

Consequently, we study the interaction of the negative externality of link specificity with the positive externality of informational value transferability. Positive and negative externalities have also been combined in one model by for instance Goyal and Joshi (2006) and Currarini (2007), but since their analysis was restricted to externalities defined on coalitions rather than precise network structure, our topology of emerging networks is new. If one reinterprets the production function as the decay factor, our approach offers an endogenous explanation of decay of transferred value. Models with endogenous decay are quite rare in the literature and have mostly been studied in the context of one-sided link formation, see Bloch and Dutta (2009), Deroian (2009), Feri and Meléndez-Jiménez (2009).

We first deal with the case of communication having social value only (Section 2) in order to isolate the impact of link specificity on network structure. When link specificity is high, the set of pairwise stable networks is characterized by two simple conditions and is shown to contain a wide range of non-standard networks, including highly connected and "small world" networks, whereas previous models for social and economic network formation without agent heterogeneity mostly predicted simple networks like stars and wheels. When link specificity is low, particular combinations of fully connected components are pairwise stable, similar to the prediction of Jackson and Wolinsky (1996) for the co-author context. Next, we deal with the case of communication from which both social and informational value is derived (Section 3 ) in order to illustrate the additional impact of value transferability on structure. Under high link specificity, only networks that consist of disjoint line components of two or three agents are shown to be pairwise stable. Apparently, the combination of high link specificity and even marginal informational value transferability has a strong fragmentizing effect on the emerging pairwise stable networks. Under low link specificity, the opposite effect takes place: already with small informational value transferability, only the complete network is pairwise stable.

Section 4 focuses on efficiency properties of the wide variety of stable networks discussed in the previous two sections. In particular, it is found that both the fragmentation under high link specificity and the dense stable networks under low link specificity are efficient in their own setting. In Section 5, link specificity values other than 1 and $1 / 2$ are investigated by simulations. Especially, it is found that 1 and $1 / 2$ are indeed suitable polar cases and that for intermediate link specificity values the common tension between stability and efficiency (e.g., Jackson and Wolinsky, 1996) is re-established. Subsequently, Section 6 concludes and offers directions for further research.

\section{Nontransferable social value}

In this section, we present a game-theoretic model for the formation of communication networks. Although we believe that communication networks typically combine social and informational value aspects, we first deal with the simpler case in which only social value is derived from communication. This approach allows us to illustrate the separate impact of link specificity on communication structure and to exclude value transferability.

Link specificity means that the more direct connections an individual has to maintain with other individuals, the less she is able to specify her attention per link. Therefore, her value per link for others declines and she also derives less value from each link with others (cf. co-author model Jackson and Wolinsky, 1996). This negative externality of link formation is crucial in our communication context, since here no benefits arise from individual contributions as such. The reason is that communication is only valuable if it is two-sided, thus effort has to be invested by both sender and receiver. ${ }^{4}$ Nevertheless, negative externalities have been largely ignored in the communication network formation literature until now. An exception are Haller and Sarangi (2005, Section 4.3), who touch on this issue in an extension of their model on heterogeneous link reliability where the addition of a link endogenously renders all adjacent links less reliable.

In short, the objective of this section is to develop a model for communication network formation with only social value from communication. We use the concept of pairwise stability to characterize the collection of stable communication networks.

\subsection{Model and stability concept}

A communication network is described by $(N, g)$, where $N=\{1, \ldots, n\}, n \geqslant 3$, is a community of agents. A direct link $g_{i j}$ between agents $i$ and $j$ in this community $(i, j \in N ; i \neq j)$ is interpreted as a communication relationship between $i$ and $j$ which is established if they both wish the link; $g_{i, j}$ indicates with a 1 or a 0 whether $i$ is directly linked to $j$ or not. These relationships are expressed by undirected links: for any two agents $i$ and $j, g_{i, j}=g_{j, i}$. By definition, $g_{i, i}=0$, as agents do not establish communication links with themselves. In this community agents only derive social value from interaction.

In case of an isolated relationship between two agents, each agent experiences social value $V^{s}>0$ as the outcome of their joint communication production process. However, maintenance of the communication relationship costs effort: investment of both agents is needed in order to make the communication specific to their personal circumstances and hence useful. Accordingly, in case of a network where two agents do not form an isolated pair, both agents are assumed to divide their effort equally among all their relationships, as a result of which, in an extreme case, the potential social communication value is divided proportionally by the number of links that agents face. However, since agents may have economies of scale in coping with several links, the extent of link specificity can be less than proportional.

We assume that the contributions of two agents in their bilateral process of communication value creation are reflected by a

\footnotetext{
${ }^{4}$ In contrast, in the co-author setting, which has been the subject of investigation in earlier research (Jackson and Wolinsky, 1996), each co-author can write independently as well.
} 
Cobb-Douglas production function with the time invested in the link by the agents as inputs. We assume both output elasticities are equal to $\rho \leqslant 1$, where $\rho=1$ corresponds to the case of high link specificity and $\rho=1 / 2$ coincides with constant returns to scale and results in low link specificity. Therefore, the total payoff for agent $i$ in communication network $g$ is given by

$$
\Pi_{i}(g)= \begin{cases}\sum_{j \in N_{i}(g)} \frac{V^{s}}{\left(\mu_{i}(g) \cdot \mu_{j}(g)\right)^{\rho}} & \text { if } \mu_{i}(g)>0, \\ 0 & \text { if } \mu_{i}(g)=0,\end{cases}
$$

where $N_{i}(g)$ is the set of agents with whom $i$ has a direct link, agent $j$ is a neighbor of agent $i$ if $j \in N_{i}(g)$, and $\mu_{i}(g)=\left|N_{i}(g)\right|$ is the number of neighbors of agent $i$, which is also referred to as the degree of $i^{5}$

For the model thus described we predict which stable networks emerge by using the concept of pairwise stability (Jackson and Wolinsky, 1996), where a network is stable if no single agent can strictly improve her payoff by deleting one of her direct links and no pair of agents can both weakly improve their payoffs by creating a direct link while at least one of the two members strictly improves her payoff by doing so. This solution concept is weak in the sense that it only assumes stability against deviations of exactly one link (which involves the permission of two agents in the case of link formation), reflecting a form of myopia. We will show that pairwise stability already clearly and interestingly constrains the number of communication networks that are stable. The study of Nash refinements in the setting of communication networks, for instance following the approach of the strongly pairwise stable set (Belleflamme and Bloch, 2004), as well as the study of farsighted stability notions in this setting, for instance following the approach of the pairwise farsightedly stable set (Herings et al., 2009) is left for future research.

In our notation, we have the following definition.

Definition 1 (pairwise stability). The network $g$ is pairwise stable if for all $i, j \in N$ with $g_{i, j}=1$ it holds that

$\Pi_{i}(g) \geqslant \Pi_{i}\left(g^{\prime}\right)$ and $\Pi_{j}(g) \geqslant \Pi_{j}\left(g^{\prime}\right)$,

where $g^{\prime}$ is such that $g_{i, j}^{\prime}=0$ and $g_{k, \ell}^{\prime}=g_{k, \ell}$ for all $\{k, \ell\} \neq\{i, j\}$, and for all $i, j \in N$ with $g_{i, j}=0$ it holds that

$\Pi_{i}(g)>\Pi_{i}\left(g^{\prime}\right)$ or $\Pi_{j}(g)>\Pi_{j}\left(g^{\prime}\right)$ or $\left(\Pi_{i}(g)=\Pi_{i}\left(g^{\prime}\right)\right.$ and $\left.\Pi_{j}(g)=\Pi_{j}\left(g^{\prime}\right)\right)$, where $g^{\prime}$ is such that $g_{i, j}^{\prime}=1$ and $g_{k, \ell}^{\prime}=g_{k, \ell}$ for all $\{k, \ell\} \neq\{i, j\}$.

\subsection{Stable networks under high link specificity}

First, we evaluate pairwise stability in communication networks under high link specificity, which we obtain by setting $\rho=1$. We prove that in this case, the collection of pairwise stable networks can be described by two easily verifiable conditions: (i) they are what we call equal neighbor degree networks, meaning that everybody has at least one neighbor and all neighbors of an agent have the same degree, and (ii) there is at most a difference of one between the degrees of agents in the same component.

Definition 2 (equal neighbor degree network). A network $g$ is an equal neighbor degree network when it holds for each $i \in N$ that $\mu_{i}(g) \geqslant 1$ and for all $j j^{\prime} \in N_{i}(g)$ that $\mu_{j}(g)=\mu_{j^{\prime}}(g)$.

For equal neighbor degree networks we will denote the own degree of an agent $i$ by $d_{i}$ and her neighbors' degree by $e_{i}$. A path in $g$ connecting $i$ and $j$ is defined as a sequence of distinct agents

\footnotetext{
${ }^{5}$ For comparison: the payoff function in the co-author model of Jackson and Wolinsky (1996) can be written as

$\Pi_{i}(g)= \begin{cases}\sum_{j \in N_{i}(g)}\left(\frac{V^{5}}{\mu_{i}(g)}+\frac{V^{5}}{\mu_{j}(g)}+\frac{V^{5}}{\mu_{i}(g) \cdot \mu_{j}(g)}\right) & \text { if } \mu_{i}(g)>0, \\ 0 & \text { if } \mu_{i}(g)=0 .\end{cases}$
}

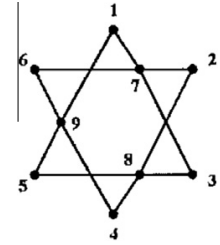

Fig. 1. An equal neighbor degree network.

$k_{1}, \ldots, k_{m} \in N$ for whom it holds that $g_{i, k_{1}}=g_{k_{1}, k_{2}}=\cdots=$ $g_{k_{m-1}, k_{m}}=g_{k_{m}, j}=1$. A component $c$ in $g$ is a network among a set of agents $C \subseteq N$ for whom it holds that for all $i, j \in C, i \neq j$, there exists a path in $c$ connecting $i$ and $j$ and $c_{i, j}=1$ if and only if $g_{i, j}=1$, and for any $i \in C$ and $j \in N \backslash C, g_{i, j}=0$. A network $g$ is a line if it has exactly $n-1$ links and there exists a sequence of distinct agents $k_{1}, \ldots, k_{n}$ $\in N$ for whom it holds that $g_{k_{1}, k_{2}}=g_{k_{2}, k_{3}}=\cdots=g_{k_{n-1}, k_{n}}=1$. A network $g$ is a star if it has exactly $n-1$ links and there exists an agent $j$ for whom it holds that $g_{j, i}=1$ for all $i \neq j$. Agent $j$ is called the center agent whereas the other agents are the periphery agents of the star.

Example 1. The network given in Fig. 1 is an equal neighbor degree network with $d_{i}=2 ; e_{i}=4$ for $i=1,2,3,4,5,6$ and $d_{i}=4$; $e_{i}=2$ for $i=7,8,9$.

Proposition 1 observes that under high link specificity, pairwise stable communication networks must be equal neighbor degree networks where the degree of two agents in the same component differs by at most one by interpreting an agent's payoff as a function of the harmonic average degree of her neighbors. ${ }^{6}$

Proposition 1. When $\rho=1$, a communication network $g$ is pairwise stable if and only if it is an equal neighbor degree network where it holds for each pair of agents $k, \ell$ in the same component that

$\left|d_{k}-d_{\ell}\right| \leqslant 1$.

Proof. Let the harmonic average degree of the neighbors of an agent $i$ with $\mu_{i}(g) \geqslant 1$ be defined as

$v_{i}(g)=\frac{\mu_{i}(g)}{\sum_{j \in N_{j}(g)} \frac{1}{\mu_{j}(g)}}$.

The payoff of agent $i$ as expressed in Eq. (1) can be written as

$\Pi_{i}(g)=\sum_{j \in N_{i}(g)} \frac{V^{s}}{\mu_{i}(g) \mu_{j}(g)}=\frac{V^{s}}{\mu_{i}(g)} \sum_{j \in N_{i}(g)} \frac{1}{\mu_{j}(g)}=\frac{V^{s}}{v_{i}(g)}$.

It is now immediate that agent $i$ strictly prefers to delete a link with agent $j$ if and only if the degree of agent $j$ is above the harmonic average degree of the neighbors of agent $i$ so if and only if

$\mu_{j}(g)>v_{i}(g)$

Similarly, and observing that the creation of a link with agent $j$ increases the degree of agent $j$ by one, agent $i$ with $\mu_{i}(g) \geqslant 1$ strictly prefers to form a link with agent $j$ if and only if

$\mu_{j}(g)+1<v_{i}(g)$.

$(\Rightarrow)$ If agent $i$ has no neighbors, then her payoff is zero, so her payoff would strictly improve by forming a link with any other agent. Any agent weakly decreases the harmonic average degree of her neighbors by forming a link with an agent who has no neighbors. Pairwise stability therefore implies $\mu_{i}(g) \geqslant 1$ for all $i \in N$.

\footnotetext{
${ }^{6}$ We are grateful to an anonymous referee for helpful suggestions to shorten the proof of Proposition 1.
} 


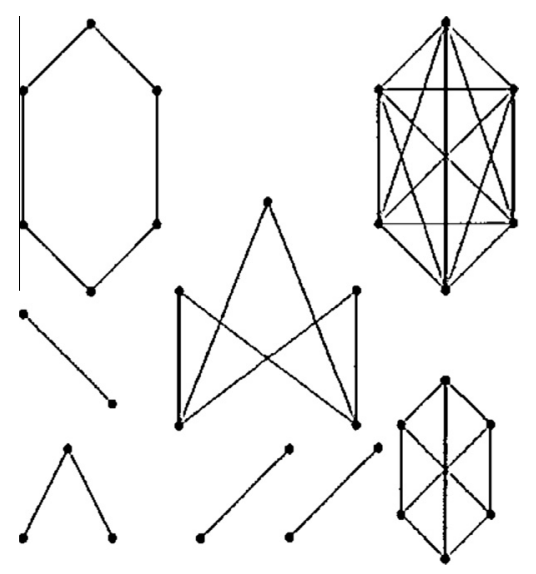

Fig. 2. A multiple-component pairwise stable communication network for $\rho=1$.

By (3) we find that all the neighbors of agent $i$ have the same degree. We have now shown that $g$ is an equal neighbor degree network.

Consider a component of $g$. If all agents in this component have the same degree, we have shown condition (2). Otherwise, using that $g$ is an equal neighbor degree network, the agents in the component can be partitioned in two groups, where within each group all agents have the same degree. We refer to these two groups as the groups of low degree and high degree agents, respectively. We show now that there are at least two low degree agents. Suppose on the contrary that agent $i$ is the only low degree agent. All neighbors of $i$ must be high degree agents and must have low degree neighbors themselves. Since their is a unique low degree agent, the neighbors of $i$ have only one neighbor themselves, so must be low degree agents, a contradiction. We have shown that there are at least two low degree agents in a component of $g$.

If the low degree differs two or more from the high degree, and since for a low degree agent $i$ it holds that $v_{i}(g)$ equals the high degree, two low degree agents would strictly prefer to form a link by (4), which would contradict pairwise stability of $g$. We have shown condition (2).

$(\Leftarrow)$ In an equal neighbor degree network, agent $i$ does not want to delete a link for then her payoff would reduce to zero if $d_{i}=1$ and would remain the same if $d_{i}>1$. Let $i, j$ be any not directly linked pair of agents, and assume without loss of generality that $d_{i} \leqslant d_{j}$. Since $e_{i} \leqslant d_{i}+1 \leqslant d_{j}+1$, it follows by (4) that creating a link with agent $j$ does not strictly improve the payoffs of agent $i$ and decreases those payoffs if one of the inequalities is strict. If all inequalities hold with equality, we have $e_{j} \leqslant d_{j}+1=d_{i}+1$, so creating a link with agent $i$ does not strictly improve the payoffs of agent $j$. It follows that being unlinked is pairwise stable for $i$ and $j$.

The following examples illustrate the wide range of networks thus proven to be pairwise stable in the social value case. ${ }^{7}$ A network $g$ is defined as complete if all agents are connected, so for all $i, j \in N$, $i \neq j$, it holds that $g_{i, j}=1$. A network $g$ is a wheel if it has exactly $n$ links and there exists a sequence of distinct agents $k_{1}, \ldots, k_{n} \in N$ for whom $g_{k_{1}, k_{2}}=g_{k_{2}, k_{3}}=\cdots=g_{k_{n-1}, k_{n}}=g_{k_{n}, k_{1}}=1$. A network $g$ is regular if it consists of one component and for all $i, j \in N$ it holds that $d_{i}=d_{j}$.

Corollary 1. When $\rho=1$, the complete, wheel, or any regular communication network is pairwise stable, for it is an equal neighbor degree network where it holds for each pair of agents $k, \ell$ in the single component that

\footnotetext{
7 This is in contrast with the case also including transferable informational value
} (Section 3.2).

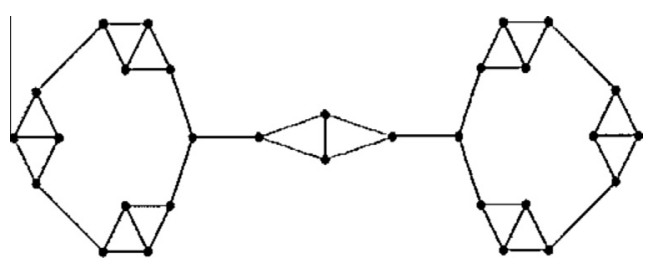

Fig. 3. A "small world" pairwise stable communication network for $\rho=1$.

$\left|d_{k}-d_{\ell}\right|=0 \leqslant 1$.

Example 2. A communication network consisting of multiple components that is pairwise stable under $\rho=1$ is given in Fig. 2 . This also exemplifies that pairwise stable networks may contain non-regular components. ${ }^{8}$

Example 3. A "small world" is a network with local clusters of highly interlinked agents together with agents that link the various clusters. As a consequence, although most agents are not directly connected, every agent is indirectly linked to every other agent by a relatively small number of steps. A regular "small world" communication network that is pairwise stable under $\rho=1$ is given in Fig. 3 .

Note that the wide set of stable communication networks includes complex real-life networks (as empirically studied by e.g. Dodds et al., 2003), whereas previous models for social and economic network formation mostly predicted simple networks like stars and wheels (e.g., Bala and Goyal, 2000; Goyal and Vega-Redondo, 2007).

If we would add a small exogenous linking cost on top of the current payoff function, the set of pairwise stable networks would be greatly reduced, and only very fragmented pairwise stable communication networks would remain, as only in an isolated pair the benefits may outweigh this cost. A similar phenomenon would occur if we would introduce some heterogeneity in $V^{s}$ such that an agent would never be indifferent between two links, and would always strictly prefer to delete one of them. As we will see in Section 3.2, the introduction of informational value surprisingly has similar fragmenting effects. At the same time, if agents would experience costs when breaking existing links, for instance caused by a status quo bias, the situation where agents maintain many links could be quite persistent.

\subsection{Stable networks under low link specificity}

In this section we study the case of low link specificity, obtained by setting $\rho=1 / 2$. We show that particular combinations of fully connected components are pairwise stable communication networks. This is similar to the prediction for the co-author model of Jackson and Wolinsky (1996), where a pairwise stable network can be partitioned into fully connected components, each of which has a different number of members: if $m_{c_{1}}$ is the number of members of one such component and $m_{c_{2}}$ is the next largest size, then $m_{c_{1}}>\left(m_{c_{2}}\right)^{2}$. We obtain a different condition because of the difference in payoff functions (see Section 2.1).

Proposition 2. Consider a communication network $g$ consisting of fully connected components $c_{1}, \ldots, c_{k}$ with $m_{c_{1}} \geqslant m_{c_{2}} \geqslant \cdots \geqslant m_{c_{k}}$, where $m_{c_{j}}$ is the number of members of $c_{j}$. When $\rho=1 / 2$ it holds that $g$ is pairwise stable if and only if $m_{c_{k}} \geqslant 2$ and $m_{c_{j}} \geqslant 4 m_{c_{j+1}}-2, j=1, \ldots$, $k-1$.

\footnotetext{
${ }^{8}$ Note that for instance any complete bipartite component where the sizes of the two sets of agents do not differ more than one satisfies the conditions of Proposition 1.
} 
Proof. We show first that $m_{c_{k}} \geqslant 2$. Since $n \geqslant 3$ it holds that $m_{c_{k}} \geqslant 3$ if $k=1$. Consider the case $k \geqslant 2$ and suppose $m_{c_{k}}=1$. A member of $c_{k-1}$ has payoff $V^{s}$, whereas creating a link with the unique member of $c_{k}$ leads to payoff

$$
\begin{aligned}
& \left(\frac{m_{c_{k-1}}-1}{\sqrt{m_{c_{k-1}}-1+1} \sqrt{m_{c_{k-1}}-1}}+\frac{1}{\sqrt{m_{c_{k-1}}-1+1}}\right) V^{\mathrm{s}} \\
& =\frac{\sqrt{m_{c_{k-1}}-1}+1}{\sqrt{m_{c_{k-1}}}} V^{\mathrm{s}},
\end{aligned}
$$

a term larger than $V^{s}$. Since obviously the unique member of $c_{k}$ wants to link with a member of $c_{k-1}$, we obtain a contradiction. Consequently, it holds that $m_{c_{k}} \geqslant 2$.

Consider a component with $m$ members. No member wants to delete a link, for the current payoff for such an agent is $V^{s}$, whereas deleting a link would reduce it to 0 when $m$ equals 2 and to

$$
\frac{m-2}{\sqrt{(m-2)(m-1)}} V^{s}
$$

when $m \geqslant 3$.

Consider two distinct components, let one component have $\ell$ members and the other $m$. A player in the $\ell$-sized component loses from establishing a link with a member of the $m$-sized component if and only if

$$
\frac{\ell-1}{\sqrt{\ell(\ell-1)}}+\frac{1}{\sqrt{\ell m}}<1
$$

which is equivalent to $1 / \sqrt{m}<\sqrt{\ell}-\sqrt{\ell-1}$, so to $\sqrt{\ell}>\sqrt{\ell-1}+1 / \sqrt{m}$. By taking squares on both sides, we obtain

$\ell>\ell-1+2 \frac{\sqrt{\ell-1}}{\sqrt{m}}+\frac{1}{m}$,

so $2 \sqrt{\ell-1} / \sqrt{m}<1-1 / m$. Taking once more squares on both sides and rearranging, we find that $m>4 \ell-2-1 / m$. Using that $m$ is an integer larger than or equal to 2 , the last expression is equivalent to $m \geqslant 4 \ell-2$.

Notice that, unless $n=7$, the collection of pairwise stable networks described in Proposition 2 contains the pairwise stable networks in the co-author model of Jackson and Wolinsky (1996). It is a subset of the collection of pairwise stable networks under high link specificity $(\rho=1$, Section 2.2$)$.

For $\rho=1 / 2$ we did not find any other pairwise stable communication networks. In particular, it is not hard to verify that neither regular communication networks with $d<n-1$ nor the example networks in Figs. 2 and 3 are stable when $\rho=1 / 2$.

\section{Informational as well as social value}

This section introduces the case in which both social and informational value is derived from communication in networks. Thus, we can illustrate the impact of value transferability on communication structure in combination with the effect of link specificity. Value transferability means that value from communication is not only derived by direct neighbors, but can also be transferred via indirect links (cf. Bala and Goyal, 2000). More specifically, we make a distinction between social and informational value derived from communication, where only informational value is transferable through the network. For example, social value from communication between two car enthusiasts only exists for the two communication partners, but informational value (e.g., from a solution to a technical problem) can exist for others in the network. After proposing a model for network formation in this setting, the pairwise stable networks are characterized again. We show that the set of stable communication networks is much more limited in range than in the purely social value setting (Section 2).

\subsection{Model}

We consider a communication network $(N, g)$ where on top of social value agents also receive informational value from interaction with other agents. In case of an isolated relationship between two agents, each agent experiences informational value $V^{i}>0$ as the outcome of their joint communication production process. Again we assume that the contributions of two agents in their bilateral process of communication value creation are reflected by a Cobb-Douglas production function with both output elasticities equal to $\rho$, where $\rho=1$ corresponds to high and $\rho=1 / 2$ to low link specificity. Agents are assumed to give relative attention to informational and social value in the proportions $\alpha$ and $1-\alpha$ respectively, where $\alpha$ is assumed to be constant satisfying $0 \leqslant \alpha \leqslant 1$.

Informational value is transferred to third parties through indirect links (paths of links), whereas social value is not transferable. This is due to the fact that in the direct communication production process of two agents, any of them can use the informational value that she acquired during the bilateral communication creation with other neighbors. Consequently, agent $j_{0}$ experiences not only first-step informational payoff from her direct neighbors:

$$
\Pi_{j_{0}}^{1 \mathrm{i}}(g)=\sum_{j_{1} \in N_{j_{0}}(g)} \frac{V^{\mathrm{i}}}{\left(\mu_{j_{0}}(g) \cdot \mu_{j_{1}}(g)\right)^{\rho}},
$$

which is similar to the social payoff in Eq. (1), but also second-step informational payoff:

$$
\Pi_{j_{0}}^{2 \mathrm{i}}(g)=\sum_{j_{1} \in N_{j_{0}}(g)} \frac{1}{\left(\mu_{j_{0}}(g) \cdot \mu_{j_{1}}(g)\right)^{\rho}} \sum_{j_{2} \in N_{j_{1}}(g) \backslash\left\{j_{0}\right\}} \frac{V^{\mathrm{i}}}{\left(\mu_{j_{1}}(g) \cdot \mu_{j_{2}}(g)\right)^{\rho}},
$$

third-step informational payoff:

$$
\begin{aligned}
\Pi_{j_{0}}^{3 \mathrm{i}}(g)= & \sum_{j_{1} \in N_{j_{0}}(g)} \frac{1}{\left(\mu_{j_{0}}(g) \cdot \mu_{j_{1}}(g)\right)^{\rho}} \sum_{j_{2} \in N_{j_{1}}(g) \backslash\left\{j_{0}\right\}} \frac{1}{\left(\mu_{j_{1}}(g) \cdot \mu_{j_{2}}(g)\right)^{\rho}} \\
& \sum_{j_{3} \in N_{j_{2}}(g) \backslash\left\{j_{1}, j_{0}\right\}} \frac{V^{\mathrm{i}}}{\left(\mu_{j_{2}}(g) \cdot \mu_{j_{3}}(g)\right)^{\rho}},
\end{aligned}
$$

and so forth, thus the overall informational payoff for $j_{0}$ is equal to

$$
\begin{aligned}
& \Pi_{j_{0}}^{\mathrm{i}}(g)=\sum_{q=1}^{n-1} \Pi_{j_{0}}^{\mathrm{qi}}(g)=V^{\mathrm{i}} \sum_{q=1}^{n-1} \prod_{r=1}^{q} \sum_{j_{r} \in N_{j_{r-1}}}(g) \backslash\left\{j_{r-2} j_{\left.r_{r-3}, \ldots . . j_{0}\right\}} \frac{1}{\left(\mu_{j_{r-1}}(g) \cdot \mu_{j_{r}}(g)\right)^{\rho}}\right.
\end{aligned}
$$

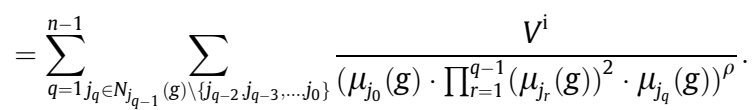

Therefore, the total payoff for agent $i$ in communication network $g$ is given by

$$
\Pi_{i}(g)= \begin{cases}\alpha \sum_{j \in \bar{N}_{i}(g)} \sum_{p \in \mathcal{P}_{i, j}(g)} \frac{v^{\mathrm{i}}}{\left(\mu_{i}(g) \cdot \prod_{k \in \bar{p}}\left(\mu_{k}(g)\right)^{2} \cdot \mu_{j}(g)\right)^{\rho}}+(1-\alpha) \sum_{j \in N_{i}(g)} \frac{V^{s}}{\left(\mu_{i}(g) \cdot \mu_{j}(g)\right)^{\rho}} & \text { if } \mu_{i}(g)>0, \\ 0 & \text { if } \mu_{i}(g)=0,\end{cases}
$$

where $\bar{N}_{i}(g)$ is the set of agents with whom $i$ has either a direct or an indirect link, $\mathcal{P}_{i, j}(g)$ is the set of paths between $i$ and $j$, and $\breve{p}$ is the set of agents on path $p$ between $i$ and $j$.

For the model thus described we again use the concept of pairwise stability (Jackson and Wolinsky, 1996) to predict which communication networks are stable. 


\subsection{Stable networks under high link specificity}

For $\rho=1$ and $0<\alpha<1$, it is proven that the pairwise stable communication networks consist of two- and three-agent line components only. ${ }^{9}$ First consider the following lemma that the star communication network becomes unstable with more than three agents.

Lemma 1. When $\rho=1$ and $0<\alpha<1$, the star communication network is pairwise stable if and only if $n=3$.

Proof. From the star network, it is not beneficial for any of the periphery agents to delete her link with the center agent as then her payoff will be zero. For the center agent, deleting a link with any of the periphery agents will provide her with the same payoff, since she is not involved in any indirect links to other agents. Periphery agent $i$ does not create a link with another periphery agent $i$ ' if and only if creating a link would not increase her payoff:

$$
\begin{aligned}
& \alpha V^{\mathrm{i}}\left(\frac{1}{n-1}+\frac{n-2}{(n-1)^{2}}\right)+(1-\alpha) V^{\mathrm{s}} \frac{1}{n-1} \\
& \quad \geqslant \alpha V^{\mathrm{i}}(\underbrace{\frac{1}{2(n-1)}+\frac{1}{8(n-1)}}_{(\mathrm{a})}+\underbrace{\frac{1}{4}+\frac{1}{4(n-1)^{2}}}_{(\mathrm{b})}+\underbrace{\frac{n-3}{2(n-1)^{2}}+\frac{n-3}{8(n-1)^{2}}}_{(\mathrm{c})}) \\
& \quad+(1-\alpha) V^{\mathrm{s}\left(\frac{1}{2(n-1)}+\frac{1}{4}\right)} \Longleftrightarrow \alpha V^{\mathrm{i}(4-n)}+(1-\alpha) V^{\mathrm{s}}(3-n) \\
& \quad \geqslant 0 \Longleftrightarrow n \leqslant 3,
\end{aligned}
$$

where the informational payoff elements on the right-hand side of the first inequality are derived from (a) the center agent, (b) agent $i$, and (c) the other periphery agents consecutively. As we assumed communities of at least three agents, it holds that $n=3$.

Now the main result can be proven by showing that when high link specificity is reinforced by value transferability, it is beneficial for agents in communication networks to break cycles and to delete links with tree branches that are longer than one link.

Proposition 3. When $\rho=1$ and $0<\alpha<1$, a communication network is pairwise stable if and only if it consists of disjoint line components of two or three agents.

\section{Proof. See Appendix A.}

Table 1 pictures all communication networks thus proven to be pairwise stable in the case with both social and informational value from communication and $\rho=1$ for $n \leqslant 6$. Comparing these results to the purely social value case (Section 2.2 ), clearly a much smaller range of very fragmented networks turns out to be pairwise stable in the mixed case where transferable informational value also plays a role. Specifically, even with $\alpha$ slightly above zero, regular communication networks are never pairwise stable and also the example networks in Figs. 2 and 3 are not stable anymore.

This may seem counter-intuitive, since apparently transferability of informational value causes networks to become more fragmented and therefore less able to transfer information. The intuition for this fragmentation is as follows. When informational value is absent, in a pairwise stable network an agent receives an equal amount of payoff from each of her links and therefore has no incentives to reduce the number of links. This is highly inefficient because of the negative externalities caused by high link

\footnotetext{
9 The results in the case where the value derived from communication is only informational $(\alpha=1)$ slightly differ from those in this mixed case $(0<\alpha<1)$. Specifically, in that case it holds that networks also containing one four-agent star component can be pairwise stable.
}

Table 1

Pairwise stable communication networks for $\rho=1$ and $\alpha>0$.

$$
\begin{array}{l|l}
n=3 \\
\hline n=4
\end{array}
$$

specificity. In the presence of informational value, agents do have incentives to break links, even if each link provides the same amount of informational value. Indeed, breaking a link with one of her neighbors in a cycle is beneficial to an agent, because it lowers the degree of that neighbor, leading to a higher informational value received from the other neighbor in the cycle. This argument shows that cycles will be absent, thereby eliminating many of the networks that were stable in the social value case. Networks without cycles are trees. Agents who are close to a leave of a tree have incentives to eliminate links with agents that are further away from the leaves. The reason is that high link specificity makes that the latter agents generate less informational value. This argument works for all trees with four or more nodes. In trees with three agents, leaves do not want to break a link in order not to become isolated. The center agent does not want to break a link, as both her neighbors are leaves, generating the same informational value.

Most studies reveal less fragmented stable networks, e.g., Goyal and Vega-Redondo (2007) find large star networks in their setting of structural holes. Therefore, our model can help to explain real-life phenomena like marriage and the evolvement of threads in online communities into strong reciprocal ties (as empirically observed by Fisher et al., 2006).

\subsection{Stable networks under low link specificity}

For $\rho=1 / 2$, we prove that the complete communication network is pairwise stable by retracing that also with value transferability it is never beneficial for an agent in the complete network to delete one of her links under this low level of link specificity.

Proposition 4. When $\rho=1 / 2$ and $0 \leqslant \alpha \leqslant 1$, the complete communication network is pairwise stable.

Proof. See Appendix A.

The following example illustrates that already at relatively low $\alpha$, multi-component communication networks (cf. Proposition 2 for $\alpha=0$ ) are not pairwise stable anymore.

Example 4. Assume $\rho=1 / 2$ and consider the communication network in Fig. 4. When $\alpha=0$, the payoff for agent $i$ is $V^{s}$ and if she would create a link with agent $k$ it would become $(1 / \sqrt{2}+1 / \sqrt{12}) V^{s} \approx 0.99578 V^{s}$. When $\alpha=1$, the payoff for $i$ is $V^{i}$ and with a link to $k$ would become $(1 / \sqrt{2}+1 / \sqrt{12}+$ $523 / 250 \sqrt{10}) V^{\mathrm{i}} \approx 1.65733 V^{\mathrm{i}}$. When $0<\alpha<1$, the payoff for $i$ is $\alpha V^{\mathrm{i}}+(1-\alpha) V^{\mathrm{s}}$ and with a link to $k$ would become

$1.65733 \alpha V^{\mathrm{i}}+0.99578(1-\alpha) V^{\mathrm{s}}$,

which for $V^{\mathrm{i}}=V^{\varsigma}$ exceeds the current payoff if $\alpha>0.0064$. Since $k$ is willing to create a link with $i$ for any $\alpha$, it holds that this network is not pairwise stable when $\alpha>0.0064$.

\section{Efficiency}

In this section, the structural results from the previous sections are assessed by their impact on efficiency. We define the efficiency 


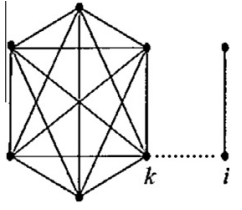

Fig. 4. A communication network that is pairwise stable when $\rho=1 / 2$ and $\alpha=0$.

of a network in terms of the sum of payoffs for all agents. ${ }^{10}$ It appears that the fragmented stable communication networks under high link specificity as well as the dense stable communication networks under low link specificity can be efficient in their own setting. Therefore, although not all pairwise stable communication networks found for $\rho=1$ and $\rho=1 / 2$ are efficient, we conclude in contrast to Jackson and Wolinsky (1996), that stability and efficiency are compatible.

Definition 3 (welfare). The welfare provided by network $g$ is given by

$W(g)=\sum_{i \in N} \Pi_{i}(g)$

Definition 4 (efficiency). The network $g$ is efficient if it holds that $W(g) \geqslant W\left(g^{\prime}\right)$ for all $g^{\prime}$.

We conjecture that for $\rho=1 / 2$, the densification that characterizes the pairwise stable communication networks has a generally beneficial influence on welfare, as well as that for $\rho=1$, the fragmentation that characterizes the pairwise stable communication networks whenever $\alpha>0$ has a generally beneficial influence on welfare. ${ }^{11}$

\section{Conjecture 1.}

(i) When $\rho=1$ and $n$ is even, a communication network consisting of disjoint pair components is efficient, and when $\rho=1$ and $n$ is odd, a communication network consisting of one three-agent line component and furthermore disjoint pair components is efficient.

(ii) When $\rho=1 / 2$, the complete communication network is efficient.

For tractability, we prove the conjecture for low $n$ and for the class of communication networks having only complete components.

Proposition 5. Conjecture 1 holds when $n=3,4,5$, or 6 .

Proof. Table 1 in the Supplementary material (Appendix B) lists the normalized welfare for $\alpha=1$ and $\alpha=0$ at $\rho=1 / 2$ and $\rho=1$ for all possible communication networks when $n=3,4,5$, and 6 , ordered according to their welfare level at $\alpha=1, \rho=1 / 2$. Given the linear combination in Eq. (5), the conjecture is hereby proven for all $0 \leqslant \alpha \leqslant 1$ and $n=3,4,5$, or 6 .

Proposition 5 shows that a three-player line is at least as efficient as a two-player line plus a singleton. For $\rho=1$ and $\alpha=0$ it is easily computed that the players in a two-player line each get a payoff of 1 , and the singleton player gets 0 . The center agent in a three-player line obtains 1 and the other two players $1 / 2$, which leads to the same welfare.

\footnotetext{
10 Alternatively, it may be interesting to study the structural effects on the actual amount of information exchanged, thus disregarding the value derived from social aspects of communication.

${ }^{11}$ For $\alpha=0$, pairwise stable communication networks tend to be overconnected, $\mathrm{cf}$. Morrill (2011) and Section 5.
}

In the presence of informational value $\alpha>0$, the three-player line has strictly higher welfare due to the indirect transfers of informational value which are now possible. In the presence of lower link specificity, $\rho<1$, the three-player line has strictly higher welfare as well.

Proposition 6. Consider the class of communication networks consisting of fully connected components $c_{1}, \ldots, c_{k}$. With $m_{c_{j}}$ the number of members of $c_{j}$, the following holds.

(i) When $\rho=1 / 2, g$ is efficient in this class when $k=1$ and $m_{c_{1}}=n$, so $g$ is complete.

(ii) When $\rho=1$ and $n$ is even, $g$ is efficient when $k=n / 2$ and $m_{c_{j}}=2$ for all $j$, so $g$ consists of disjoint pairs. ${ }^{12}$

Proof. Because of symmetry in a complete component it suffices to consider the payoffs for one member and because of the linear combination in Eq. (5) it suffices to consider the cases $\alpha=0$ and $\alpha=1$.

(i) For $\rho=1 / 2$ and $\alpha=0$, the normalized payoff for an agent in component $c_{j}$ is 0 when $m_{c_{j}}=1$ and 1 when $m_{c_{j}} \geqslant 2$. For $\rho=1 / 2$ and $\alpha=1$, the normalized payoff for an agent in component $c_{j}$ is 0 when $m_{c_{j}}=1,1$ when $m_{c_{j}}=2$, and

$$
1+\sum_{q=2}^{m_{c_{j}}-1} \frac{\prod_{r=2}^{q}\left(m_{c_{j}}-r\right)}{\left(m_{c_{j}}-1\right)^{q-1}}=1+\sum_{q=2}^{m_{c_{j}}-1} \prod_{r=2}^{q} \frac{m_{c_{j}}-r}{m_{c_{j}}-1}
$$

when $m_{c_{j}} \geqslant 3$, which is $3 / 2$ for $m_{c_{j}}=3$ and increasing in $m_{c_{j}}$.

(ii) For $\rho=1$ and $\alpha=0$, the normalized payoff for an agent in component $c_{j}$ is 0 when $m_{c_{j}}=1$ and $1 /\left(m_{c_{j}}-1\right)$ when $m_{c_{j}} \geqslant 2$, which is 1 for $m_{c_{j}}=2$ and decreasing in $m_{c_{j}}$. For $\rho=1$ and $\alpha=1$, the normalized payoff for an agent in component $c_{j}$ is 0 when $m_{c_{j}}=1,1$ when $m_{c_{j}}=2$, and

$$
\frac{1}{m_{c_{j}}-1}+\sum_{q=2}^{m_{c_{j}}-1} \frac{\prod_{r=2}^{q}\left(m_{c_{j}}-r\right)}{\left(m_{c_{j}}-1\right)^{2 q-1}}=\frac{1+\sum_{q=2}^{m_{c_{j}}-1} \prod_{r=2}^{q} \frac{m_{c_{j}}-r}{\left(m_{c_{j}}-1\right)^{2}}}{m_{c_{j}}-1}
$$

when $m_{c_{j}} \geqslant 3$, which is $5 / 8$ for $m_{c_{j}}=3$ and decreasing in $m_{c_{j}}$.

\section{Other values of link specificity}

In this section, other link specificity values than 1 and $1 / 2$ are investigated by simulations of our communication network formation model. Because of the wide variety of possible pairwise stable and efficient networks for each $\rho$ value (cf. Sections 2-4), simulations are considered to be a pragmatic tool to provide insight into effects of gradual changes in $\rho$. They illustrate that 1 and $1 / 2$ are indeed suitable polar cases and that for intermediate values the common tension between stability and efficiency (e.g., Jackson and Wolinsky, 1996) is re-established.

\subsection{Method}

\subsubsection{Pairwise stability}

A simulation starts with a randomly generated network, where for every $i, j \in N$ with $i \neq j$, the link $g_{i, j}$ is formed with probability $1 / 2$.

In every iteration we randomly determine whether there will be an attempt to delete or create a link. If this turns out to be delete, one agent is drawn from the community and subsequently another one. If there exists a link between these two agents, the first agent

\footnotetext{
12 Notice that for odd $n$, the most efficient communication network as predicted by
} Conjecture 1 includes a component that is not complete. 
calculates the payoff she will earn when she deletes this link (Eq. (5)). If this is strictly higher than the payoff she earns with the current network, the link is deleted. If no link exists between the two agents or the first agent does not gain by its deletion, the current network is maintained until the next iteration.

If the attempt appears to be create, two agents are randomly drawn from the community. If no link exists between these two agents, they both calculate the payoff they will earn when they create such a link. If this is weakly higher for both agents and strictly higher for at least one of them, the link is created. If there already exists a link between these two agents or one of them loses or none of them gains by its creation, the current network is maintained.

In this way, 5000 iterations are performed consecutively. Afterwards it is verified whether the simulation converged to a pairwise stable network. We perform 500 of these simulations for each of 45 specifications of parameter values, with $\rho$ equal to $0.1,0.2, \ldots, 1.5$ and $\alpha$ equal to $0,1 / 2,1$.

\subsubsection{Pairwise efficiency}

We also perform simulations to compute pairwise efficient communication networks. This is achieved by repeating the procedure of Section 5.1.1, assuming that agents all hold welfare as their objective function (Definition 3). A network is said to be pairwise efficient if no pair of agents can improve welfare.

Definition 5 (pairwise efficiency). The network $g$ is pairwise efficient if for all $i, j \in N$ with $g_{i, j}=1$ it holds that

$W(g) \geqslant W\left(g^{\prime}\right)$,

where $g^{\prime}$ is such that $g_{i, j}^{\prime}=0$ and $g_{k, l}^{\prime}=g_{k, l}$ for all $\{k, l\} \neq\{i, j\}$, and for all $i, j \in N$ with $g_{i, j}=0$ it holds that

$W(g) \geqslant W\left(g^{\prime}\right)$,

where $g^{\prime}$ is such that $g_{i, j}^{\prime}=1$ and $g_{k, l}^{\prime}=g_{k, l}$ for all $\{k, l\} \neq\{i, j\}$.

Clearly, an efficient network is pairwise efficient, though the reverse is not necessarily the case.

\subsubsection{Further specifications}

Community size $n=6$ is chosen for all simulations, since it is large enough to illustrate interesting tendencies as well as small enough to generate reasonable calculation times regarding the exponentially increasing number of paths in the payoff function (Eq. (5)). Furthermore, we take $V^{i}=V^{s}=6$ because of symmetry and since values of 6 are convenient when computing the payoffs in specific examples. We have verified that our results are robust for the case with an odd number of agents $n=5$.

In order to compare simulation outcomes among levels of $\rho$ and $\alpha$, we use the density of a network (Wasserman and Faust, 1994, p. 164):

$D(g)=\frac{\sum_{i=1}^{n} \mu_{i}(g)}{n(n-1)}=\frac{1}{30} \sum_{i=1}^{6} \mu_{i}(g)$.

For example, the empty network has density 0 , a network consisting of three disjoint pair components has density 0.2 , a network consisting of two disjoint three-agent line components has density 0.27 , a network consisting of a four-agent wheel component and a disjoint pair component or a network consisting of a three-agent complete component and a disjoint three-agent line component has density 0.33 , a wheel network or a network consisting of two disjoint complete three-agent components has density 0.4 , a network consisting of a complete four-agent component and a disjoint pair component has density 0.47 , a regular network with degree 3 has density 0.6 , a regular network with degree 4 has density 0.8 , and the complete network has density 1 .
Table 2

Simulated effect of $\rho$ on density of pairwise stable and pairwise efficient communication networks for $n=6$ and $\alpha=0,1 / 2,1$.

\begin{tabular}{|c|c|c|c|c|c|c|}
\hline \multirow[t]{2}{*}{$\rho \backslash \alpha$} & \multicolumn{2}{|l|}{0} & \multicolumn{2}{|l|}{$1 / 2$} & \multicolumn{2}{|l|}{1} \\
\hline & pws & pwe & pws & pwe & pws & pwe \\
\hline 0.1 & 1.00 & 1.00 & 1.00 & 1.00 & 1.00 & 1.00 \\
\hline 0.2 & 1.00 & 1.00 & 1.00 & 1.00 & 1.00 & 1.00 \\
\hline 0.3 & 1.00 & 1.00 & 1.00 & 1.00 & 1.00 & 1.00 \\
\hline 0.4 & 1.00 & 1.00 & 1.00 & 1.00 & 1.00 & 1.00 \\
\hline 0.5 & 1.00 & 0.58 & 1.00 & 1.00 & 1.00 & 1.00 \\
\hline 0.6 & 1.00 & 0.22 & 1.00 & 0.43 & 1.00 & 0.52 \\
\hline 0.7 & 0.95 & 0.22 & 1.00 & 0.25 & 1.00 & 0.40 \\
\hline 0.8 & 0.70 & 0.23 & 0.69 & 0.23 & 0.54 & 0.22 \\
\hline 0.9 & 0.38 & 0.23 & 0.37 & 0.24 & 0.36 & 0.24 \\
\hline 1.0 & 0.27 & 0.23 & 0.23 & 0.24 & 0.23 & 0.24 \\
\hline 1.1 & 0.20 & 0.20 & 0.20 & 0.23 & 0.20 & 0.24 \\
\hline 1.2 & 0.20 & 0.20 & 0.20 & 0.20 & 0.20 & 0.23 \\
\hline 1.3 & 0.20 & 0.20 & 0.20 & 0.20 & 0.20 & 0.20 \\
\hline 1.4 & 0.20 & 0.20 & 0.20 & 0.20 & 0.20 & 0.20 \\
\hline 1.5 & 0.20 & 0.20 & 0.20 & 0.20 & 0.20 & 0.20 \\
\hline
\end{tabular}

Our intuitive prediction is that in general, a higher level of link specificity $\rho$ makes communication more costly, and therefore the density of a simulated communication network lower. This intuition is confirmed by the analytically found potential outcomes for the cases $\rho=1$ and $\rho=1 / 2$ (Sections 2 and 3). A higher level of focus on informational value $\alpha$ provides more value spillovers from indirect links, and thus the expected density of a pairwise stable communication network is higher. As noted at the end of Section 3.2 , this intuition is not confirmed by the analytically found potential outcomes: whereas in Section 2.2 a large range of possibly dense communication networks is proven to be pairwise stable for $\rho=1$ and $\alpha=0$, in Section 3.2 only very fragmented communication networks turn out to be pairwise stable for $\rho=1$ and $\alpha=1$.

\subsection{Results}

It appears that all 500 simulations in every setting converge to pairwise stable networks as described in Section 5.1.1. Therefore, we deal with density and welfare of these networks.

\subsubsection{Density}

For each combination of 15 levels of link specificity $\rho$ and three levels of focus on informational versus social value from communication $\alpha$, the average density of the 500 simulated pairwise stable and pairwise efficient networks is given in Table 2 and represented in Fig. 5.

The basic intuition about the effect of $\rho$ on density in communication networks is thus confirmed by the simulation outcomes as it was by the analytical results of Sections 2 and 3, as the density of the pairwise stable networks is generally decreasing in $\rho$. Notice that this also roughly holds for the pairwise efficiency simulation outcomes, although a few small exceptions appear, for example at $\rho=0.8$.

The intuition about the effect of $\alpha$ on density in communication networks is again contradicted by the simulation outcomes, e.g., for $\rho=0.8$ we find a higher average density for $\alpha=1 / 2(0.69)$ than for $\alpha=1(0.54)$, and for $\rho=1.0$ we find a higher average density for $\alpha=0(0.27)$ than for $\alpha=1 / 2(0.23) .{ }^{13}$ Notice that our intuition about the effect of $\alpha$ on density in communication networks is still confirmed by the pairwise efficiency simulation outcomes, in particular for $\rho$ around 0.6 .

\footnotetext{
13 Thus, for $\alpha=0$ the simulation process on average selects relatively sparse networks from the wide range of pairwise stable networks.
} 


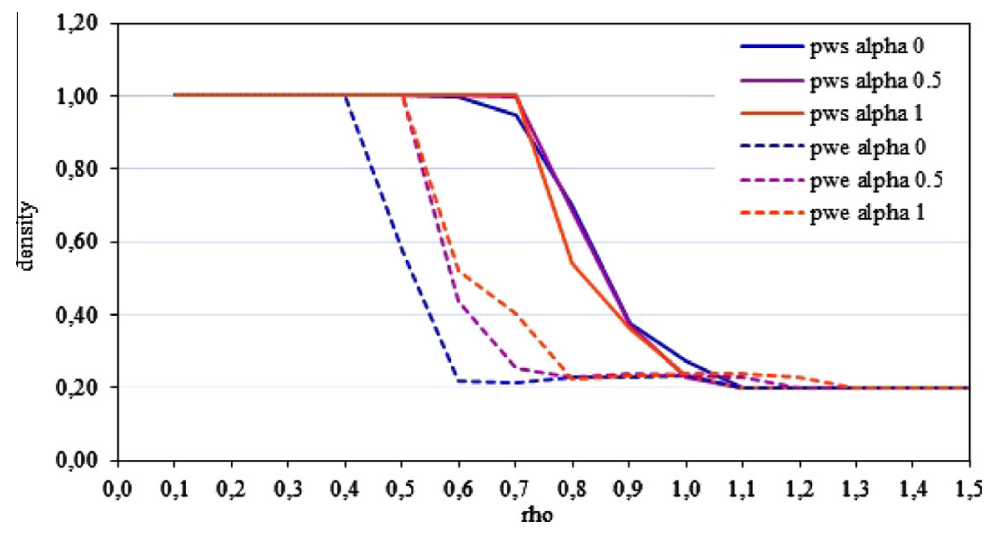

Fig. 5. Simulated effect of $\rho$ on density of pairwise stable and efficient communication networks for $n=6$ and $\alpha=0,0.5,1$.

Table 3

Example of simulated communication networks.

pairwise stable networks pairwise efficient networks

The simulations confirm that $\rho=1$ and $\rho=1 / 2$ are suitable polar cases, since outside these boundaries the simulated pairwise stable as well as efficient communication networks largely coincide with either the complete network or rather a network consisting of pairs only, whereas in between we find large differences in density between the pairwise efficient and pairwise stable communication networks, where pairwise stable networks are more connected than pairwise efficient networks. Thus, for intermediate levels of $\rho$, the pairwise stable communication networks are highly connected as with lower levels of link specificity, whereas the pairwise efficient communication networks are very fragmented as with higher levels of link specificity. The following example elaborates such a case.

Example 5. Let $\rho=3 / 4, \alpha=1 / 2$. Table 3 provides an overview of the types and numbers of pairwise stable communication networks resulting from 100 simulations and the types and numbers of pairwise efficient communication networks resulting from another 100 simulations.

\subsubsection{Efficiency}

As a means to investigate to what extent the density differences between the pairwise efficient and pairwise stable communication networks actually lead to welfare differences, the average welfare provided by the 500 simulated pairwise stable and pairwise efficient networks is given in Table 4 and represented in Fig. 6 for each parameter setting. For comparison, the expected welfare from a random network ${ }^{14}$ and the maximum welfare have also been calculated for each setting.

Although we have seen in Section 5.2.1 that a higher $\alpha$ and thus more value transferability does not always lead to more dense communication networks, it apparently does always lead to higher

\footnotetext{
14 This was based on 5000 networks generated in the same way as the start networks for the simulations.
}

welfare, as the welfare of the pairwise stable networks is increasing in $\alpha$, even strictly so for $\rho \leqslant 1.0 .^{15}$

Furthermore, though a lower $\rho$ has been shown to lead to higher density in communication networks, it only leads to subsequent higher welfare when link specificity is low enough, since for intermediate values of $\rho$, welfare is much lower and partly even strictly increases in $\rho$. Thus, the welfare of a community is higher for low and high values of $\rho$ than for intermediate ones.

Moreover, we find that the density difference between pairwise stability and pairwise efficiency in communication networks found for $\alpha=0, \rho=0.5$ does not lead to a subsequent welfare difference, but for intermediate levels of link specificity $(1 / 2<\rho<1)$, the tension between pairwise efficiency and pairwise stability is considerable (up to larger than $40 \%$ for $\alpha=0$ and $\rho=0.7,0.8$ ). In the most extreme intervals (i.e., around $\rho=0.7$ ), the simulated pairwise stable networks on average even provide less welfare than a random network. Note that this effect is due to overconnection of the type illustrated in Table 3.

Interestingly, on the other hand a few parameter combinations exist where social preferences appear to be harmful for welfare in the sense that the simulated pairwise efficient communication networks are not globally optimal, whereas pairwise stability does lead to maximum welfare $(\alpha=1 / 2, \rho=1.1$ and $\alpha=1, \rho=1.1,1.2)$. Most of this is due to situations where the centers of two threeagent line components do not want to delete one of their links, because this would in the first instance harm welfare, whereas pairwise stability would lead both of them to delete one of their links, after which the two isolated agents can form a new pair, which increases welfare in the second instance.

\section{Discussion}

This paper has shown that the structure of bilateral communication links within communication networks can be appropriately studied using a model based on the game-theoretic literature of social and economic network formation. A combination of important aspects common to communication networks was incorporated that had not been investigated until now: the negative externality of link specificity, which was neglected in the communication network context so far, and the positive externality of informational value transferability.

In the case of communication having nontransferable social value only (Section 2), illustrating the separate impact of link specificity on structure, the set of pairwise stable communication

\footnotetext{
15 Notice that this claim does depend on the assumption $V^{\mathrm{i}}=V^{\varsigma}$ chosen for the simulations. For example, it can be shown that for $\rho=1$ and $V^{\mathrm{s}}=x V^{\mathrm{i}}, \alpha$ has an opposite effect on efficiency if $x>5 / 4$
} 
Table 4

Simulated effect of $\rho$ on welfare of pairwise stable, pairwise efficient, random, and optimal communication networks for $n=6$ and $\alpha=0$, $1 / 2,1$.

\begin{tabular}{|c|c|c|c|c|c|c|c|c|c|c|c|c|}
\hline \multirow[t]{2}{*}{$\rho \backslash \alpha$} & \multicolumn{4}{|l|}{0} & \multicolumn{4}{|l|}{$1 / 2$} & \multicolumn{4}{|l|}{1} \\
\hline & pws & pwe & rand & $\max$ & pws & pwe & rand & $\max$ & pws & pwe & rand & $\max$ \\
\hline 0.1 & 130.5 & 130.5 & 73.4 & 130.5 & 1758.8 & 1758.8 & 274.3 & 1758.8 & 3387.2 & 3387.2 & 471.8 & 3387.2 \\
\hline 0.2 & 94.6 & 94.6 & 59.9 & 94.6 & 601.3 & 601.3 & 160.3 & 601.3 & 1108.1 & 1108.1 & 258.8 & 1108.1 \\
\hline 0.3 & 68.5 & 68.5 & 49.1 & 68.5 & 243.0 & 243.0 & 101.8 & 243.0 & 417.4 & 417.4 & 154.3 & 417.4 \\
\hline 0.4 & 49.7 & 49.7 & 40.4 & 49.7 & 115.8 & 115.8 & 70.0 & 115.8 & 181.8 & 181.8 & 99.2 & 181.8 \\
\hline 0.5 & 36.0 & 35.9 & 33.4 & 36.0 & 63.2 & 63.2 & 50.4 & 63.2 & 90.4 & 90.4 & 67.3 & 90.4 \\
\hline 0.6 & 26.1 & 34.8 & 27.6 & 36.0 & 38.0 & 42.6 & 37.8 & 43.0 & 50.0 & 54.6 & 48.0 & 55.4 \\
\hline 0.7 & 19.7 & 34.5 & 23.0 & 36.0 & 24.5 & 35.9 & 29.3 & 36.0 & 29.9 & 43.6 & 35.7 & 43.6 \\
\hline 0.8 & 19.3 & 32.2 & 19.2 & 36.0 & 22.1 & 33.8 & 23.2 & 36.0 & 30.1 & 35.7 & 27.2 & 36.0 \\
\hline 0.9 & 23.9 & 31.6 & 16.1 & 36.0 & 26.8 & 32.0 & 18.7 & 36.0 & 30.0 & 33.6 & 21.3 & 36.0 \\
\hline 1.0 & 29.2 & 30.2 & 13.5 & 36.0 & 32.3 & 31.0 & 15.2 & 36.0 & 32.9 & 32.2 & 17.0 & 36.0 \\
\hline 1.1 & 36.0 & 36.0 & 11.5 & 36.0 & 36.0 & 31.3 & 12.5 & 36.0 & 36.0 & 31.1 & 13.7 & 36.0 \\
\hline 1.2 & 36.0 & 36.0 & 9.7 & 36.0 & 36.0 & 36.0 & 10.5 & 36.0 & 36.0 & 31.8 & 11.2 & 36.0 \\
\hline 1.3 & 36.0 & 36.0 & 8.3 & 36.0 & 36.0 & 36.0 & 8.8 & 36.0 & 36.0 & 36.0 & 9.4 & 36.0 \\
\hline 1.4 & 36.0 & 36.0 & 7.1 & 36.0 & 36.0 & 36.0 & 7.5 & 36.0 & 36.0 & 36.0 & 7.9 & 36.0 \\
\hline 1.5 & 36.0 & 36.0 & 6.1 & 36.0 & 36.0 & 36.0 & 6.4 & 36.0 & 36.0 & 36.0 & 6.7 & 36.0 \\
\hline
\end{tabular}

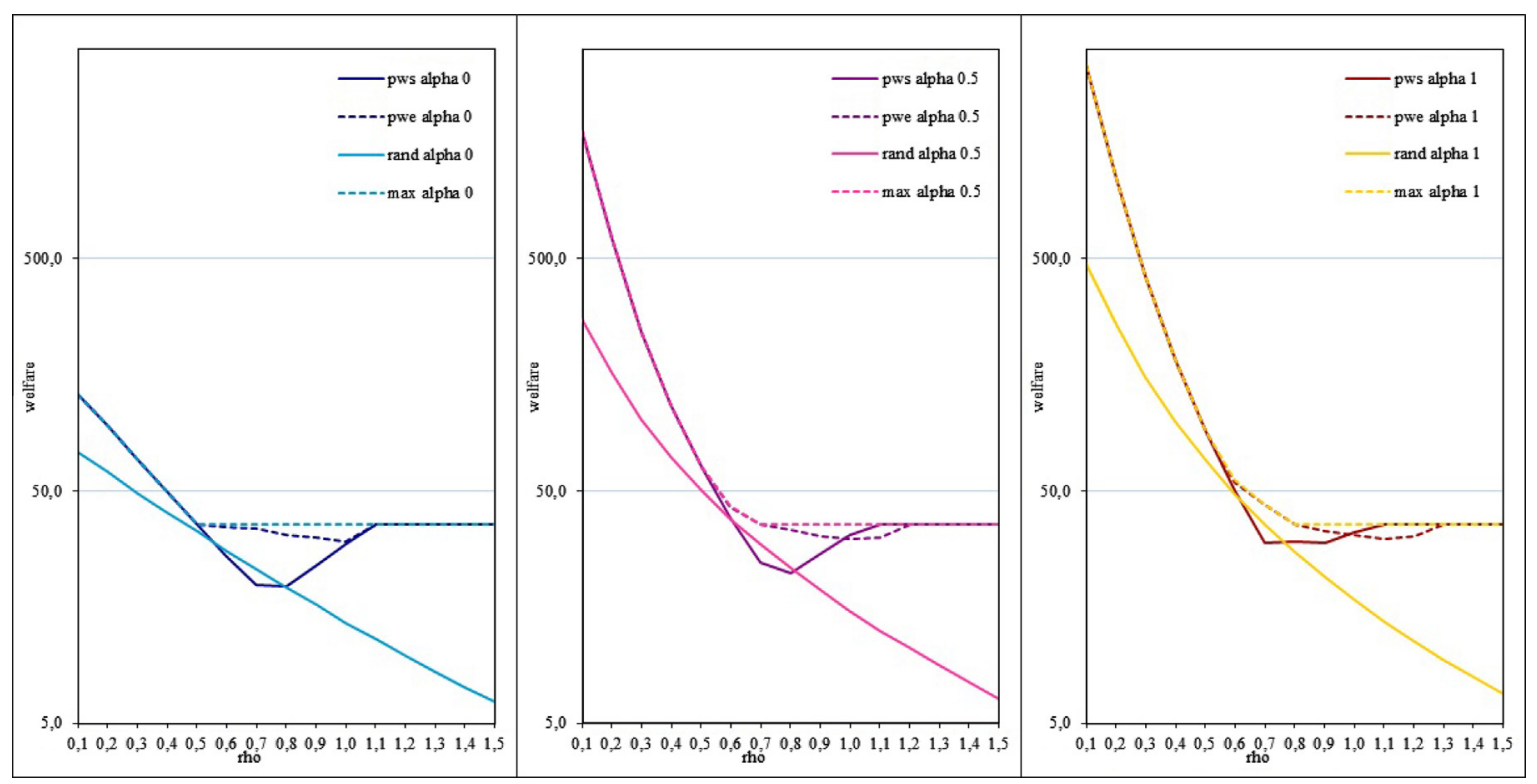

Fig. 6. Simulated effect of $\rho$ on welfare of pairwise stable, pairwise efficient, random, and optimal communication networks for $n=6$ and $\alpha=0$, $0.5,1$.

networks was characterized for high link specificity and shown to include a wide range of non-standard networks like highly connected and "small world" networks, whereas previous models for social and economic network formation without agent heterogeneity mostly predicted simple networks like stars and wheels. For low link specificity, particular combinations of fully connected components were proven to be pairwise stable communication networks in line with the co-author model of Jackson and Wolinsky (1996).

In the case of communication from which both social and informational value is derived (Section 3), illustrating the joint impact of link specificity and value transferability on structure, under high link specificity only networks that consist of disjoint line components of two or three agents were shown to be pairwise stable. Herewith, we predict much more fragmentation than usually in the literature about social and economic network formation, where mostly only either of these two features was included. Under low link specificity, the opposite extreme effect takes place: already with small informational value transferability, multi-component communication networks may fail to be pairwise stable.
Both the fragmentation under high link specificity and the dense pairwise stable communication networks under low link specificity are efficient in their own setting (Section 4), whereas for intermediate link specificity values quite inefficient communication networks may arise (Section 5).

Intuition predicts that a higher level of link specificity $\rho$ makes communication more costly, and therefore stable communication networks will be sparser. This is confirmed by the analytical results for the cases $\rho=1$ and $\rho=1 / 2$ (Sections 2 and 3) as well as by the simulation outcomes for other $\rho$ values (Section 5). However, lower link specificity and thus higher density only leads to subsequent higher welfare when link specificity is low enough, since for intermediate values of $\rho$, welfare is much lower (Section 5). This is caused by the fact that from a welfare point of view pairwise stable networks are too dense for intermediate values of $\rho$ and the negative externalities associated to link specificity. This implies that enhancing communication in the community by decreasing link specificity from a high to an intermediate level results in lower welfare from communication. These results suggest that communities should either go for low link specificity or for high link specificity in their communication efforts. Notice that link specificity 
can often be influenced by communities, since it is affected by the choice of communication technology and (in-)formal rules of conduct such as bureaucratic hurdles and conventions on communication style.

A higher level of focus on transferable informational value $\alpha$ provides more positive value spillovers from indirect links, so intuition predicts higher density for pairwise stable communication networks. This is contradicted by the analytical results for $\rho=1$ (Sections 2 and 3 ) and to a smaller extent by the simulation outcomes for relatively high $\rho$ values (Section 5), which can be ascribed to the interaction effect of value transferability and high link specificity. However, when the potential social and informational value present in a community are equal, higher $\alpha$ does always lead to higher welfare, even strictly so for $\rho \leqslant 1.0$ (Section 5). Therefore, stimulating the focus on informational value of communication can be a generally effective tool for boosting welfare. In line with intuition, this effectivity is larger the smaller $\rho$.

Future studies could introduce agent heterogeneity, e.g., valuation heterogeneity in the sense that individuals represent different values for their fellows or have different opinions on the values of their fellows, like Galeotti et al. (2006) for standard communication network formation models. For example, when $\rho=1$ and $0<\alpha<1$, if we assume a valuation pattern deviating from full homogeneity in the sense that there is one agent $j$ who is valued differently than all other agents, it can be proven that all pairwise stable communication networks consist of small line components and one possibly larger component without cycles containing the differing agent $j$ but not at the periphery. In particular, this component may be a star component with agent $j$ at the center.

Another extension of the current model could be to relax the assumption that agents divide their available effort equally among all their relationships, thus increasing the variability of link strength by dropping the common one-zero formulation of links. Bloch and Dutta (2009) started such a task mainly for a setting with one-sided link formation and found rather similar results as in their discrete variant. However, this similarity is partly ascribed to the assumption of a convex relationship between individual effort and link strength because of a fixed cost of link formation, whereas in our model a linear relationship would be more in line with the assumed costs of link maintenance rather than formation.

Besides, a possible follow-up would be to empirically examine the applicability of the used payoff function in diverse contexts. The model could be tested experimentally, contributing to an emerging literature as surveyed by Kosfeld (2004). Accordingly, we hope that our current work stimulates future research in the appealing area of communication networks and the roles of affecting link specificity as well as balancing nontransferable social and transferable informational value.

\section{Acknowledgements}

The authors gratefully acknowledge two anonymous referees for their valuable suggestions and helpful comments. The second author would like to thank the Netherlands Organisation for Scientific Research (NWO) for financial support.

\section{Appendix A}

Proof of Proposition 3. $(\Leftarrow)$ It is not beneficial for any of the periphery agents in a two- or three-agent line component to delete her single link as then her payoff will be zero. Equivalently, for the center agent in a three-agent line component, deleting a link with any of the two periphery agents is not beneficial as it will provide her with the same payoff.
Lemma 1 implies that the periphery agents of a three-agent line do not create links. Therefore, we only have to examine the following cases $(a)-(f)$ related to link formation between two agents in different components:

\begin{tabular}{llll}
\hline & $\begin{array}{l}\text { Pair } \\
\text { agent }\end{array}$ & $\begin{array}{l}\text { Center agent } \\
\text { of 3-agent line }\end{array}$ & $\begin{array}{l}\text { Periphery agent } \\
\text { of 3-agent line }\end{array}$ \\
\hline $\begin{array}{c}\text { Pair agent } \\
\begin{array}{c}\text { Center agent of } \\
\text { 3-agent line }\end{array}\end{array}$ & $\mathrm{x}$ & $($ b $)$ & $($ c $)$ \\
$\begin{array}{c}\text { Periphery agent } \\
\text { of 3-agent } \\
\text { line }\end{array}$ & $\mathrm{x}$ & $\mathrm{x}$ & $(e)$ \\
\hline
\end{tabular}

For each of these cases, it can be proven by evaluating the payoffs with and without the link that no link is created: after forming a link in case $(a)$, a pair agent would get payoff

$\alpha V^{\mathrm{i}}\left(\frac{1}{2}+\frac{1}{4}+\frac{1}{8}\right)+(1-\alpha) V^{\mathrm{s}}\left(\frac{1}{2}+\frac{1}{4}\right) \leqslant \alpha V^{\mathrm{i}}+(1-\alpha) V^{\mathrm{s}}$,

after forming a link in case $(b)$, the pair agent would get payoff

$\alpha V^{\mathrm{i}}\left(\frac{1}{2}+\frac{1}{6}+\frac{1}{18}+\frac{1}{18}\right)+(1-\alpha) V^{\mathrm{s}}\left(\frac{1}{2}+\frac{1}{6}\right)<\alpha V^{\mathrm{i}}+(1-\alpha) V^{\mathrm{s}}$,

after forming a link in case $(c)$, the pair agent would get payoff $\alpha V^{\mathrm{i}}\left(\frac{1}{2}+\frac{1}{4}+\frac{1}{16}+\frac{1}{32}\right)+(1-\alpha) V^{\mathrm{s}}\left(\frac{1}{2}+\frac{1}{4}\right)<\alpha V^{\mathrm{i}}+(1-\alpha) V^{\mathrm{s}}$, after forming a link in case $(d)$, a center agent would get payoff

$$
\begin{aligned}
& \alpha V^{\mathrm{i}}\left(\frac{1}{3}+\frac{1}{3}+\frac{1}{9}+\frac{1}{27}+\frac{1}{27}\right)+(1-\alpha) V^{\mathrm{s}}\left(\frac{1}{3}+\frac{1}{3}+\frac{1}{9}\right) \\
& \leqslant \alpha V^{\mathrm{i}}\left(\frac{1}{2}+\frac{1}{2}\right)+(1-\alpha) V^{\mathrm{s}}\left(\frac{1}{2}+\frac{1}{2}\right),
\end{aligned}
$$

after forming a link in case $(e)$, the center agent would get payoff

$$
\begin{aligned}
& \alpha V^{\mathrm{i}}\left(\frac{1}{3}+\frac{1}{3}+\frac{1}{6}+\frac{1}{24}+\frac{1}{48}\right)+(1-\alpha) V^{\mathrm{s}}\left(\frac{1}{3}+\frac{1}{3}+\frac{1}{6}\right) \\
& <\alpha V^{\mathrm{i}}\left(\frac{1}{2}+\frac{1}{2}\right)+(1-\alpha) V^{\mathrm{s}}\left(\frac{1}{2}+\frac{1}{2}\right),
\end{aligned}
$$

and after forming a link in case $(f)$, a periphery agent would get payoff

$$
\begin{aligned}
& \alpha V^{\mathrm{i}}\left(\frac{1}{4}+\frac{1}{4}+\frac{1}{8}+\frac{1}{16}+\frac{1}{32}\right)+(1-\alpha) V^{\mathrm{s}}\left(\frac{1}{4}+\frac{1}{4}\right) \\
& \leqslant \alpha V^{\mathrm{i}}\left(\frac{1}{2}+\frac{1}{4}\right)+(1-\alpha) V^{\mathrm{s}} \frac{1}{2} .
\end{aligned}
$$

$(\Rightarrow)$ For this part of the proof, we need some extra notation. The payoff function in (5) can be rewritten as

$\Pi_{i}(g)=\frac{1}{\mu_{i}(g)} \sum_{j \in N_{i}(g)} T_{i, j}(g)$,

where $T_{i, j}(g)$ is the total payoff that $j$ transfers to $i$ via her direct link with $i$. Formally,

$$
\begin{aligned}
T_{i, j}(g)= & \alpha\left(\frac{V^{\mathrm{i}}}{\mu_{j}(g)}+\sum_{\left(j^{\prime} \in \overline{\left.N_{j}(g) \backslash\{i\}\right)}\right.} \sum_{\left(p \in \mathcal{P}_{j j^{\prime}}(g): i \notin \breve{p}\right)} \frac{V^{\mathrm{i}}}{\mu_{j^{\prime}}(g) \cdot\left(\mu_{j}(g)\right)^{2} \cdot \prod_{k \in \breve{p}}\left(\mu_{k}(g)\right)^{2}}\right) \\
& +(1-\alpha) \frac{V^{s}}{\mu_{j}(g)} .
\end{aligned}
$$

Assume that $g$ is a pairwise stable network. Let $i$ be an agent in $g$ and $k \in N_{i}(g)$ be such that 
$T_{i, k}(g)=\min _{j \in N_{i}(g)} T_{i, j}(g)$.

Suppose that there exists an agent $\ell \in N_{i}(g)$ for whom it holds that $T_{i, \ell}(g)>T_{i, k}(g)$.

Deleting the link between $i$ and $k$ results in network $g^{\prime}$, where it holds that

$T_{i, j}\left(g^{\prime}\right) \geqslant T_{i, j}(g), \forall j \in N_{i}\left(g^{\prime}\right)$,

since $k$, to whom $j$ might be (in)directly linked, has one costly direct link less, so more informational value might flow from $j$ to $i$ via $k$. The payoff for $i$ then becomes

$\Pi_{i}\left(g^{\prime}\right)=\frac{1}{\mu_{i}(g)-1} \sum_{j \in N_{i}\left(g^{\prime}\right)} T_{i, j}\left(g^{\prime}\right)>\frac{1}{\mu_{i}(g)} \sum_{j \in N_{i}(g)} T_{i, j}(g)=\Pi_{i}(g)$,

which contradicts pairwise stability of $g$. It follows that

$T_{i, j}(g)=T_{i, j^{\prime}}(g), \forall j, j^{\prime} \in N_{i}(g)$.

Next, suppose that $g$ contains a cycle, meaning that there exists a sequence of distinct agents $k_{1}, \ldots, k_{n} \in N$ for whom it holds that $g_{k_{1}, k_{2}}=g_{k_{2}, k_{3}}=\cdots=g_{k_{n-1}, k_{n}}=g_{k_{n}, k_{1}}=1$. Let $i$ be an agent in this cycle. Deleting the link with one of $i$ 's neighbors in the cycle, say $k$, results in $g^{\prime}$, where it holds for the other neighbor of $i$ in the cycle, say $m$, that

$T_{i, m}\left(g^{\prime}\right)>T_{i, m}(g)$,

since $k$, to whom $m$ is (in)directly linked, has one costly direct link less, so more informational value flows from $k$ to $i$ via $m$. Moreover,

$T_{i, j}\left(g^{\prime}\right) \geqslant T_{i, j}(g), \forall j \in N_{i}\left(g^{\prime}\right)$.

The payoff for $i$ then becomes

$$
\begin{aligned}
\Pi_{i}\left(g^{\prime}\right) & =\frac{1}{\mu_{i}(g)-1} \sum_{j \in N_{i}\left(g^{\prime}\right)} T_{i, j}\left(g^{\prime}\right)>\frac{1}{\mu_{i}(g)-1} \sum_{j \in N_{i}\left(g^{\prime}\right)} T_{i, j}(g) \\
& =\frac{1}{\mu_{i}(g)} \sum_{j \in N_{i}(g)} T_{i, j}(g)=\Pi_{i}(g),
\end{aligned}
$$

where the second equality follows from Eq. (6). This implies that $g$ is not pairwise stable, leading to a contradiction. We have therefore shown that $g$ does not contain any cycle.

Since we have already shown that $g$ contains no cycles, all components of $g$ are trees. In a tree the number of links is one less than the number of agents. Moreover, in a tree there is a unique path between any two agents. A tree that is not a star contains an agent, say $i$, with a neighbor $h$ that only has $i$ as a neighbor, and, moreover, $i$ is directly linked to an agent $j$ who has another neighbor different from $i$. According to Eq. (6) it holds that

$T_{i, h}(g)=T_{i, j}(g)$.

Since $h$ has only one neighbor, $i$, it follows that

$T_{i, h}(g)=\alpha V^{\mathrm{i}}+(1-\alpha) V^{\mathrm{s}}$.

We now evaluate $T_{i, j}(g)$ and show it is smaller than $T_{i, h}(g)$.

Think of the component $\bar{N}_{h}(g)$ to which $h$ belongs as a tree with $h$ as top agent. For players $k, k^{\prime} \in \bar{N}_{h}(g), k \neq k^{\prime}$, player $k^{\prime}$ is a subordinate of $k$, denoted $k^{\prime} \in \bar{S}(k)$, if $k$ is on the unique path from $h$ to $k^{\prime}$. Player $k^{\prime}$ is a direct subordinate of $k$, denoted $k^{\prime} \in S(k)$, if $k^{\prime}$ is a subordinate of $k$ and there is a link between $k$ and $k^{\prime}$. We write

$T_{i, j}(g)=\alpha T_{i, j}^{\mathrm{i}}(g)+(1-\alpha) T_{i, j}^{\mathrm{s}}(g)$

where

$T_{i, j}^{\mathrm{s}}(g)=\frac{V^{\mathrm{s}}}{\mu_{j}(g)} \leqslant \frac{V^{\mathrm{s}}}{2}$, and

$T_{i, j}^{\mathrm{i}}(g)=\frac{V^{\mathrm{i}}}{\mu_{j}(g)}+\sum_{k \in \bar{S}(j)} \frac{V^{\mathrm{i}}}{\mu_{k}(g)\left(\mu_{j}(g)\right)^{2} \prod_{k^{\prime} \in \breve{p}_{j, k}}\left(\mu_{k^{\prime}}(g)\right)^{2}}$,

where $p_{j, k}$ is the unique path between $j$ and $k$.

Consider $k \in \bar{S}(i)$. We define the informational payoff that $k$ receives from its subordinates by

$U_{k}^{\mathrm{i}}(g)=\frac{1}{\mu_{k}(g)} \sum_{k^{\prime} \in S(k)} T_{k, k^{\prime}}^{\mathrm{i}}(g)$

where $T_{k, k^{\prime}}^{\mathrm{i}}(\mathrm{g})$ is defined analogously to $T_{i, j}^{\mathrm{i}}(\mathrm{g})$. We obtain a recursive relation by noting that

$T_{k, k^{\prime}}^{\mathrm{i}}(g)=\frac{V^{\mathrm{i}}+U_{k^{\prime}}^{\mathrm{i}}(g)}{\mu_{k^{\prime}}(g)}$.

We show by induction that

$U_{k}^{\mathrm{i}}(g) \leqslant V^{\mathrm{i}}\left(\mu_{k}(g)-1\right)$,

from which it follows that

$T_{k, k^{\prime}}^{\mathrm{i}}(g) \leqslant \frac{V^{\mathrm{i}}+V^{\mathrm{i}}\left(\mu_{k^{\prime}}(g)-1\right)}{\mu_{k^{\prime}}(g)}=V^{\mathrm{i}}$,

and, consequently,

$T_{i, j}^{\mathrm{i}}(g) \leqslant V^{\mathrm{i}}$.

Let $K^{0} \subset \bar{N}_{h}(g)$ be the set of agents without subordinates. For $m \geqslant 1$, let $K^{m}$ be the set of agents with all subordinates in $K^{0}$ $\cup \cdots \cup K^{m-1}$. Let $m^{\prime}$ be the smallest integer for which $j \in K^{m^{\prime}}$. First consider an agent $k$ in $K^{0}$, the set of agents without subordinates. Then $U_{k}^{\mathrm{i}}(g)=0=V^{\mathrm{i}}\left(\mu_{k}(g)-1\right)$, so (9) is satisfied.

Suppose that (9) holds for agents in $K^{m}, m<m^{\prime}$. Consider an agent $k \in K^{m+1}$.

$$
\begin{aligned}
U_{k}^{\mathrm{i}}(g) & =\frac{1}{\mu_{k}(g)} \sum_{k^{\prime} \in S(k)} T_{k, k^{\prime}}^{\mathrm{i}}(g) \leqslant \frac{1}{\mu_{k}(g)} \sum_{k^{\prime} \in S(k)}\left(\frac{V^{\mathrm{i}}+V^{\mathrm{i}}\left(\mu_{k^{\prime}}(g)-1\right)}{\mu_{k^{\prime}}(g)}\right) \\
& =\frac{\mu_{k}(g)-1}{\mu_{k}(g)} V^{\mathrm{i}} \leqslant \frac{1}{2} V^{\mathrm{i}}\left(\mu_{k}(g)-1\right),
\end{aligned}
$$

so (9) holds for all $k \in \bar{S}(i)$.

Combining (8) and (10) implies $T_{i, j}(g)<T_{i, h}(g)$, a contradiction to Eq. (7), so $g$ consists of star components only.

The proof of Lemma 1 implies that these stars have at most three agents. Stars of a single agent cannot be part of $g$, for it is always strictly beneficial for this single agent to create a link to the center agent of another star, whereas this center agent is indifferent or improves if she is isolated too. "Stars" of two or three agents are lines.

Proof of Proposition 4. We normalize payoffs by setting $V^{\mathrm{i}}=1$. For $\alpha=1$, the payoff for an agent in the complete network is

$1+\sum_{q=2}^{n-1} \frac{\prod_{r=2}^{q}(n-r)}{(n-1)^{q-1}}$

where $q$ indicates the step level, and if she deletes a link her payoff becomes

$\frac{1+2(n-2) \frac{\sqrt{n-2}}{\sqrt{n-1}}}{n-1}+\sum_{q=3}^{n-1} \frac{\prod_{r=3}^{q}(n-r)}{(n-1)^{q-1}}\left(1+\frac{n^{2}-5 n+q+4}{\sqrt{n-1} \sqrt{n-2}}\right)$,

where the first term combines payoffs resulting from paths with lengths 1 and 2. Subtracting the latter from the former gives

$\sum_{q=3}^{n-1}\left(\frac{\prod_{r=3}^{q}(n-r)}{(n-1)^{q-1}}\left(n-3-\frac{n^{2}-5 n+q+4}{\sqrt{n-1} \sqrt{n-2}}\right)+\frac{2(n-2)-2(n-2) \frac{\sqrt{n-2}}{\sqrt{n-1}}}{(n-1)(n-3)}\right)$. 
We have to prove that (11) is nonnegative. Multiplying by $(n-1)$, we find that it is sufficient to show that

$$
\begin{aligned}
& \sum_{q=3}^{n-1}\left(\left(\prod_{r=3}^{q} \frac{n-r}{n-1}\right)\left(n-3-\frac{n^{2}-5 n+q+4}{\sqrt{n-1} \sqrt{n-2}}\right)+\frac{2(n-2)}{n-3}\right. \\
& \left.-\frac{2(n-2)^{2}}{(n-3) \sqrt{n-1} \sqrt{n-2}}\right) \geqslant 0 .
\end{aligned}
$$

When we define

$$
\begin{aligned}
& a(q)=\prod_{r=3}^{q} \frac{n-r}{n-1}, \\
& b(q)=n-3-\frac{n^{2}-5 n+q+4}{\sqrt{n-1} \sqrt{n-2}},
\end{aligned}
$$

then the first term in (12) is given by

$$
\sum_{q=3}^{n-1} a(q) b(q) \text {. }
$$

The second minus the third term in (12) is positive. We show by means of contradiction that the first term is nonnegative. Suppose the first term is negative, implying $n \geqslant 4$.

Notice that $a(q) \geqslant 0$ and $b(q)$ is decreasing in $q$, so there is $\bar{q} \geqslant 3$ such that $3 \leqslant q<\bar{q}$ implies $a(q) b(q) \geqslant 0$ and $\bar{q} \leqslant q \leqslant n-1$ implies $a(q) b(q)<0$. This fact together with the supposition

$$
\sum_{q=3}^{n-1} a(q) b(q)<0
$$

implies that

$$
\sum_{q=3}^{n-1} a(q) b(q)>\sum_{q=3}^{n-1} \lambda(q) a(q) b(q)
$$

for coefficients $\lambda(q)$ larger than or equal to 1 and nondecreasing in $q$. We define

$\lambda(q)=\prod_{r=3}^{q-1} \frac{n-1}{n-r}$,

with $\lambda(3)=1$ by definition. Then we have obtained the desired contradiction once we show that

$\sum_{q=3}^{n-1}\left(\frac{n-q}{n-1}\left(n-3-\frac{n^{2}-5 n+q+4}{\sqrt{n-1} \sqrt{n-2}}\right)+\frac{2(n-2)}{n-3}-\frac{2(n-2)^{2}}{(n-3) \sqrt{n-1} \sqrt{n-2}}\right) \geqslant 0$.

It holds that

$$
\begin{aligned}
& \sum_{q=3}^{n-1} \frac{n-q}{n-1}(n-3)=\frac{(n-2)(n-3)^{2}}{2(n-1)}, \\
& \sum_{q=3}^{n-1} \frac{n-q}{n-1} \frac{n^{2}-5 n+4}{\sqrt{n-1} \sqrt{n-2}}=\frac{(n-2)(n-3)(n-4)}{2 \sqrt{n-1} \sqrt{n-2}},
\end{aligned}
$$

$$
\begin{aligned}
& \sum_{q=3}^{n-1} \frac{n-q}{n-1} \frac{q}{\sqrt{n-1} \sqrt{n-2}}=\frac{n(n-3)(n+2)}{2(n-1) \sqrt{n-1} \sqrt{n-2}}-\frac{2 n^{3}-3 n^{2}+n-30}{6(n-1) \sqrt{n-1} \sqrt{n-2}}, \\
& \sum_{q=3}^{n-1} \frac{2(n-2)}{n-3}-\frac{2(n-2)^{2}}{(n-3) \sqrt{n-1} \sqrt{n-2}}=2(n-2)-\frac{2(n-2)^{2}}{\sqrt{n-1} \sqrt{n-2}},
\end{aligned}
$$

where for the third inequality we use the fact that $1^{2}+2^{2}+\cdots+r^{2}=\frac{1}{3} r^{3}+\frac{1}{2} r^{2}+\frac{1}{6} r$. After multiplying by 6 and rewriting we obtain the inequality

$$
\frac{3 n^{3}-12 n^{2}+27 n-30}{n-1}-\frac{3 n^{4}-17 n^{3}+45 n^{2}-73 n+54}{(n-1) \sqrt{n-1} \sqrt{n-2}} \geqslant 0 .
$$

The expression on the left-hand side exceeds

$$
\frac{3 n^{3}-12 n^{2}+27 n-30}{n-1}-\frac{3 n^{4}-17 n^{3}+45 n^{2}-73 n+54}{(n-1)\left(n-\frac{8}{5}\right)} \text {. }
$$

Cross multiplying, we find that the last expression is larger than or equal to zero if and only if

$$
\begin{gathered}
3 n^{4}-16 \frac{4}{5} n^{3}+46 \frac{1}{5} n^{2}-73 \frac{1}{5} n+48 \\
\geqslant 3 n^{4}-17 n^{3}+45 n^{2}-73 n+54 .
\end{gathered}
$$

For $n \geqslant 4$, such is clearly the case. Thus, the complete network is pairwise stable for $\alpha=1$.

Since it follows from Proposition 2 that the complete network is stable for $\alpha=0$ and given the linear combination in Eq. (5), the complete network is pairwise stable for $0 \leqslant \alpha \leqslant 1$.

\section{Appendix B. Supplementary material}

Supplementary data associated with this article can be found in the online version, at http://dx.doi.org/10.1016/j.ejor.2013.02.028.

\section{References}

Asvanund, A., Clay, K., Krishnan, R., Smith, M.D., 2004. An empirical analysis of network externalities in peer-to-peer music-sharing networks. Information Systems Research 15, 155-174.

Bala, V., Goyal, S., 2000. A noncooperative model of network formation. Econometrica 68, 1181-1229.

Baron, R., Durieu, J., Haller, H., Solal, P., 2006. Complexity and stochastic evolution of dyadic networks. Computers \& Operations Research 33, 312-327.

Belleflamme, P., Bloch, F., 2004. Market sharing agreements and collusive networks. International Economic Review 45, 387-411.

Bloch, F., Dutta, B., 2009. Communication networks with endogenous link strength. Games and Economic Behavior 66, 39-56.

Campbell, J.F., 1994. Integer programming formulations of discrete hub location problems. European Journal of Operational Research 72, 387-405.

Currarini, S., 2007. Network design in games with spillovers. Review of Economic Design 10, 305-326.

Deroian, F., 2009. Endogenous link strength in directed communication networks. Mathematical Social Sciences 57, 110-116.

Dodds, P.S., Muhamad, R., Watts, J.W., 2003. An experimental study of search in global social networks. Science 301, 827-829.

Feri, F., Meléndez-Jiménez, M.A., 2009. Coordination in Evolving Networks with Endogenous Decay. University of Innsbruck Working Papers in Economics and Statistics, vol. 19

Fisher, D., Smith, M., Welser, H.T., 2006. You are who you talk to: detecting roles in Usenet newsgroups. In: Proceedings of the 39th Hawaii International Conference on System Sciences, vol. 3. p. 59.2.

Galeotti, A., 2010. Talking, searching, and pricing. International Economic Review 51, 1159-1174.

Galeotti, A., Goyal, S., Kamphorst, J., 2006. Network formation with heterogeneous players. Games and Economic Behavior 54, 353-372.

Goyal, S., Joshi, S., 2006. Unequal connections. International Journal of Game Theory 34, 319-349.

Goyal, S., Vega-Redondo, F., 2007. Structural holes in social networks. Journal of Economic Theory 137, 460-492.

Granovetter, M., 2005. The impact of social structure on economic outcomes. Journal of Economic Perspectives 19, 33-50.

Haller, H., Sarangi, S., 2005. Nash networks with heterogeneous agents. Mathematical Social Sciences 50, 181-201.

Hancock, P.G., Raeside, R., 2010. Analysing communication in a complex service process: an application of social network analysis in the Scottish Prison Service. Journal of the Operational Research Society 61, 265-274.

Herings, P.J.J., Mauleon, A., Vannetelbosch, V., 2009. Farsightedly stable networks. Games and Economic Behavior 67, 526-541.

Jackson, M.O., Wolinsky, A., 1996. A strategic model of social and economic networks. Journal of Economic Theory 71, 44-74.

Janssen, R.H.P., Monsuur, H., 2012. Stable network topologies using the notion of covering. European Journal of Operational Research 218, 755-763.

Kelly, F.P., Maulloo, A.K., Tan, D.K.H., 1998. Rate control for communication networks: shadow prices, proportional fairness and stability. Journal of the Operational Research Society 49, 237-252.

Kosfeld, M., 2004. Economic networks in the laboratory: a survey. Review of Network Economics 3, 20-41.

Mathwick, C., Wiertz, C., De Ruyter, K., 2008. Social capital production in a virtual P3 community. Journal of Consumer Research 34, 832-849.

Monsuur, H., 2007. Stable and emergent network topologies: a structural approach. European Journal of Operational Research 183, 432-441.

Morrill, T., 2011. Network formation under negative degree-based externalities. International Journal of Game Theory 40, 367-385.

Trier, M., 2008. Towards dynamic visualization for understanding evolution of digital communication networks. Information Systems Research 19, 335-350.

Wasserman, S., Faust, K., 1994. Social Network Analysis: Methods and Applications. University Press, Cambridge. 